\title{
Comparative Study of the Comprehensive Properties of Multiwalled Nanotubes/Polyurethane Nanofibrous Membranes with and without Thermal Treatment
}

\author{
Ling Liu ${ }^{1}$ and Shengqiang Pan ${ }^{2}$ \\ ${ }^{1}$ School of Aerospace Engineering \& Applied Mechanics, Tongji University, Shanghai 200092, China \\ ${ }^{2}$ School of Civil Engineering and Mechanics, Central South University of Forestry and Technology, Changsha 410004, China
}

Correspondence should be addressed to Ling Liu, lingliu@tongji.edu.cn

Received 3 December 2011; Revised 5 January 2012; Accepted 19 January 2012

Academic Editor: Tong Lin

Copyright (C) 2012 L. Liu and S. Pan. This is an open access article distributed under the Creative Commons Attribution License, which permits unrestricted use, distribution, and reproduction in any medium, provided the original work is properly cited.

\begin{abstract}
This paper makes use of electrospinning technology to produce multiwalled nanotubes and polyurethane (MWNTs/PU) composite nanofibrous membranes. A thermal treatment method was proposed to improve the comprehensive properties of the membranes. The morphology of the prepared nanomaterials was characterized by the SEM and TEM. Comprehensive properties, including thermal stability, conductivity, and mechanical properties, of the membranes with and without thermal treatment were investigated comparatively by TGA, DMA, resistivity measurement, and tensile testing, respectively. The results indicate that the proposed thermal treatment method could effectively improve the conductivity and mechanical properties of the nanomaterials. For the thermal treatment-based membrane filled with $3 \mathrm{wt}$ \% of MWNTs, the conductivity reaches $8.29 \times 10^{-5} \mathrm{Scm}^{-1}$, which is nearly three orders higher than that of the untreated sample. Meanwhile, the tensile strength and modulus increase more than $50 \%$ after thermal treatment. It is expected that such improved nanomaterials may be potentially useful in composite reinforcement and electronic devices.
\end{abstract}

\section{Introduction}

Compared with more traditional fibers such as carbon and glass fibers, nanofibers have several specific features such as smaller diameter, high-surface area per unit mass, high strength-to-weight ratio, and ability in providing both mechanical as well as functional properties $[1,2]$. Thus, nanofibers have attracted considerable attention in recent years. Typically, nanofibers are prepared by drawing, template synthesis, self-assembly, emulsion polymerization, chemical vapor deposition, and electrospinning [3]. Among the various manufacturing technologies, electrospinning process is a simple, feasible, and economical means to fabricate nanofibers. Nanofibers prepared by electrospinning technology can be found applications in coatings, membranes, fabrics, and scaffolds [3, 4].

The main weakness of these nanofibers obtained by electrospinning seems to be their poor mechanical properties caused by relaxation processes occurring immediately after fiber formation, at which a certain degree of molecular orientation is lost $[5,6]$. To functionalize and improve the physical properties of fibers, several types of nanoparticles have been studied. Among them, carbon nanotubes (CNTs) have received a particular attention [20-23]. Due to their rolled graphite structure, CNTs have superior in-plane properties, for example, Young's modulus is $1 \mathrm{Tpa}$, and the resistivity is $50 \mu \Omega \mathrm{cm}^{2}[7,8]$. In fact, after their rapid development, CNTs have been widely used to improve electrical or mechanical properties of electrospun polymer nanofibers [9-14].

In addition, the nature of CNT structure enables their surfaces to be modified chemically with various functional groups, as demonstrated by a handful of research groups $[15,16]$. Studies have also highlighted that addition of CNTs can increase crystallinities and change crystal morphologies of the polymer matrix $[12,17]$. Moreover, the presence of CNTs enhances the conductivity of the polymersolution and produces a larger electrical current during electrospinning $[18,19]$. The addition of charge accumulation overcomes 
cohesive forces and intensifies repulsive forces among the charges accumulated inside nanofibers. Therefore, the CNTs within the polymeric solution would align and disperse themselves along the fiber axis during the spinning process, and fibers with smaller diameter and well-dispersed CNTs are formed $[12,18,19]$. Due to afore-mentioned advantages, relative studies reported that incorporating of CNTs into the nanofibers could averagely improve the electric conductivity and tensile strength of the nanofibrous membrane by 10 orders and 2-to-4 times, respectively [5-17, 20-23].

Although, remarkable physical properties have been achieved by the use of this method, bulk mechanical properties of the membrane remain relatively low [5-17, 20-23]. This is because a large number of nanofibers are randomly stacked one over another to form the membrane, in which, one fiber is independent of another. It means that the membrane in nature is a discontinuous stress transmission carrier, which results a poor mechanical property. So, this work aims to improve the properties of the membrane by a simple thermal treatment method, expecting a continuous mesh membrane can be obtained.

Polyurethane (PU) is a resilient elastomer that has a wide range of application as coatings, sealants, transdermal patches, and biomaterials such as catheters and bloodcontacting environments [24]. In the present work, electrospinning technique was used to produce multi-walled nanotubes(MWNTs) and thermal polyurethane (PU) composite nanofibrous membranes. A thermal treatment method is proposed to obtain a membrane with better comprehensive properties. Morphology, thermal properties, electric conductivity, and mechanical properties of the MWNTs/PU membranes with and without thermal treatment were examined by SEM, TEM, TGA, DMA, resistivity measurement, and tensile testing, respectively. Results have shown that the thermal treatment method can further enhance the properties of the membranes, especially the conductivity and mechanical properties.

\section{Experimental}

2.1. Materials. MWNTs (purchased from Shenzhen Nanotech Port Co., Ltd., China) were modified with carboxyl, which were functionalized by the manufacturer and with a carboxyl ratio of $2.3 \mathrm{wt} . \%$. The carboxylic MWNTs is $10 \sim$ $20 \mathrm{~nm}$ in diameter, $5 \sim 15 \mu \mathrm{m}$ in length, and purity is greater than 95\%. Thermoplastic PU (BASF, 1185A) was dissolved in tetrahydrofuran (THF) and $N-N$ dimethylformamide (DMF) (THF: DMF $=6: 4$ by weight) at $80^{\circ} \mathrm{C}$ for 6 hours. A prepared solution suitable for electrospinning had a total PU concentration of about $10 \mathrm{wt} . \%$.

\subsection{Preparation}

2.2.1. Electrospinning. The dispersion of MWNTs in PU solution was prepared with help of a sonicator (model KQ118, $70 \mathrm{~W}$, China) for $3-4 \mathrm{~h}$ at $60^{\circ} \mathrm{C}$ to obtain homogeneous dispersion. The dispersions were directly used in the electrospinning process. The electrospinning set used in this
TABLE 1: Samples prepared for characterization.

\begin{tabular}{lcc}
\hline Sample & Concentration of MWNTs (wt.\%) & Thermal treatment \\
\hline A & 0 & No \\
A $^{\prime}$ & 0 & Yes \\
B & 1 & No \\
B $^{\prime}$ & 1 & Yes \\
C & 3 & No \\
C $^{\prime}$ & 3 & Yes \\
\hline
\end{tabular}

research was described in [25]. Electrospinning was carried out at room temperature in air with an injection speed of $3.0 \sim 4.0 \mathrm{~mL} / \mathrm{h}$, a high electric voltage of $23 \sim 24 \mathrm{kV}$, and a distance between electrodes of $13 \sim 14 \mathrm{~cm}$, respectively. The nanofibers/nanowebs were received on the surface of aluminum foil with a random direction, and the thickness of the obtained nanowebs membrane was in the range of $80-100 \mu \mathrm{m}$. The concentration of MWNTs was varied 0,1 , and 3 wt.\%, with respect to the solution mixture (shown in Table 1).

2.2.2. Thermal Treatment. A thermal processing was applied to the electrospun nanofibers (shown in Table 1) in web form to take advantages of dispersing MWNTs in the nanofibers. Firstly, the nanofibrous membranes were put into a vacuum drying chamber, a piece of release film was put on the upper side of the membrane, then covered with a steel plate to pressurize the membranes. After closing the door of the chamber, a vacuum was created to the chamber, and the temperature inside the chamber was increased to $95^{\circ} \mathrm{C}$ at a rate of $1-2^{\circ} \mathrm{C} / \mathrm{min}$ and held at $95-105^{\circ} \mathrm{C}$ for 5 6 hours in a vacuum status. Finally, removed the vacuum and cooled down the temperature to room temperature. The thermal processing was expected to increase the structural stability of the nanofibrous membranes, thereby enabling the fabrication of membranes with well-dispersed MWNTs and continuous mesh.

\subsection{Characterization}

2.3.1. Morphology. The final morphologies and surface structures of the nanofibers with various MWNTs loadings were observed with a SEM (Philips XL 30 ESEM instrument). Fiber diameters within the membrane were measured using software ImageJ. TEM (HITACHI H-800) was used to confirm that MWNTs were embedded in the nanofibers and aligned along the nanofibers axis. The samples with and without thermal treatment were observed for a comparison purpose.

2.3.2. Thermal Analysis. TG analysis was performed for all the samples using a NETZSCH Instruments STA 449C in nitrogen gas, at a heat rate of $20^{\circ} \mathrm{C} / \mathrm{min}$, from ambient to $600^{\circ} \mathrm{C}$. DMA was operated on a TA Instruments Q800 in nitrogen gas (at low temperature), operating in the tensile test mode at an oscillation frequency of $1 \mathrm{~Hz}$. Data were 


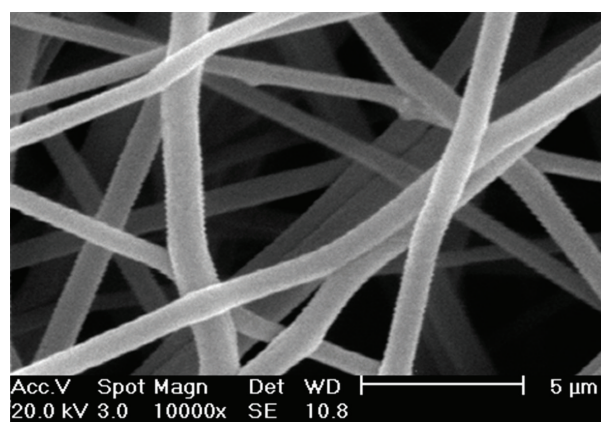

(A)

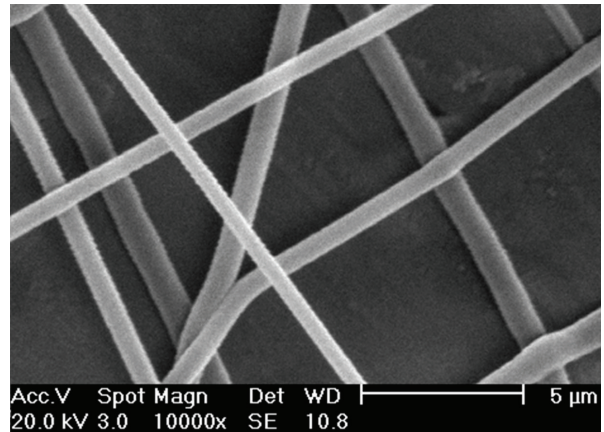

(B)

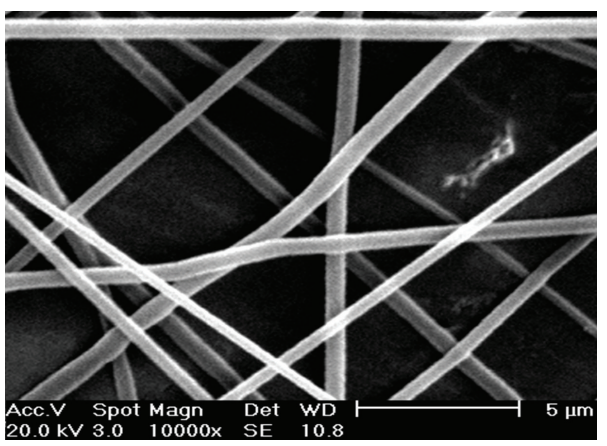

(C)

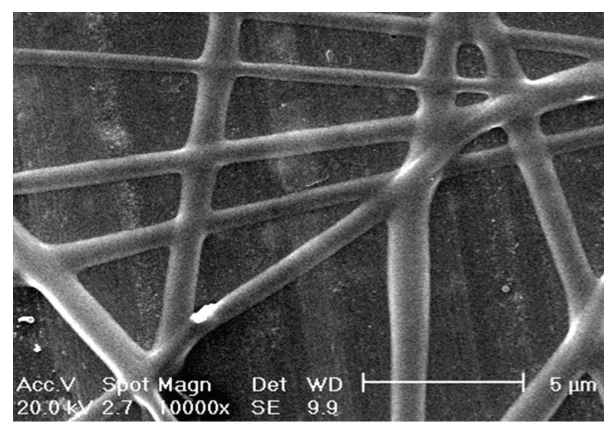

$\left(\mathrm{A}^{\prime}\right)$

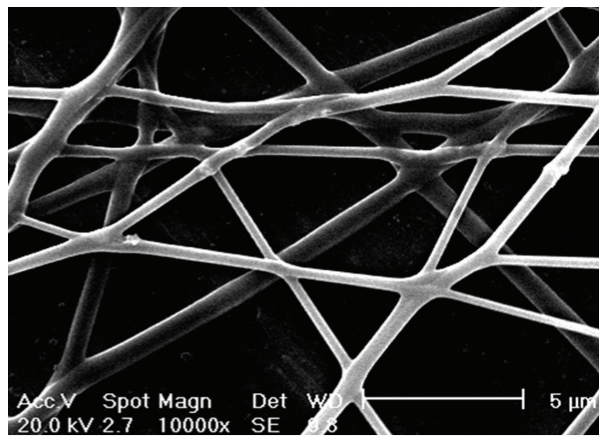

$\left(\mathrm{B}^{\prime}\right)$

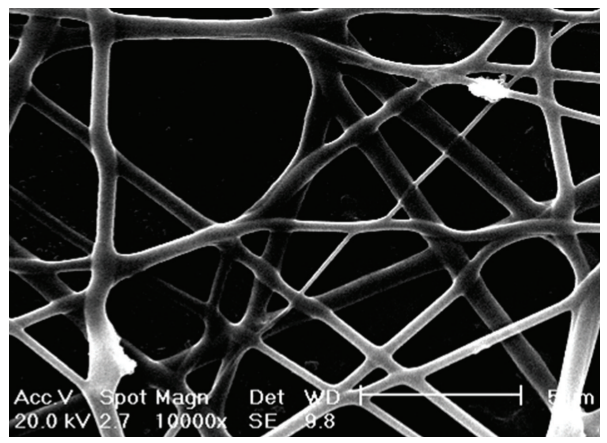

$\left(\mathrm{C}^{\prime}\right)$

Figure 1: SEM images of MWNTs/PU nanofibers without thermal treatment $(\mathrm{A}, \mathrm{B}$, and $\mathrm{C})$ and with thermal treatment $\left(\mathrm{A}^{\prime}, \mathrm{B}^{\prime}\right.$, and $\left.\mathrm{C}^{\prime}\right)$.

collected from $-110 \sim 100^{\circ} \mathrm{C}$ at a scanning rate of $5^{\circ} \mathrm{C} / \mathrm{min}$. The sample specimens were cut into rectangular bars with dimensions of $60 \mathrm{~mm} * 10 \mathrm{~mm} *(0.08-0.10) \mathrm{mm}$.

2.3.3. Electrical Conductivity. Electrical conductivity of all final membranes was measured using various instruments. In the case of high-resistance specimens $\left(>10^{7} \Omega\right)$, a PC68 (Shanghai Precision Instrument Company, China), highresistance meter was used, in a three-electrode mode, and in the low-resistance range $\left(<10^{7} \Omega\right)$ a Loresta GP resistance meter was used in a four-probe mode. The conductivities obtained from above methods are direct-current (DC) conductivities, which can be calculated using the measured resistance and the geometry of the samples.

The effective alternating-current (AC) conductivities of the specimens were measured using an Agilent 4294A impedance analyzer in the frequency range of $40 \mathrm{~Hz}$ to $110 \mathrm{MHz}$ with an AC voltage of $500 \mathrm{mV}$. Complex impedance modulus, $Z$, of all samples at different frequencies can be obtained from the analyzer. Therefore, AC conductivity, $\sigma$, can be calculated from the formula: $\sigma=(1 / Z) *(t / A)$, where $t$ is thickness of the membranes, and $A$ is the area of the electrode. The two end surfaces of all samples were coated with conductive adhesive for ohmic contact.

2.3.4. Mechanical Properties. To characterize the tensile performance of the nanofibrous membranes, a special clamping fixture was used [25], which was fixed on a universal testing machine (Model CSS-44020, Changchun Institute of Testing Machines, China), equipped with a $10 \mathrm{~N}$ or $20 \mathrm{~N}$ load cell. All of the test specimens were cut to $50 \mathrm{~mm}$ long and $10 \mathrm{~mm}$ wide. Each end was affixed with two pieces of end tapes of $10 \mathrm{~mm}$ length on both the sides, leaving a gauge length of $30 \mathrm{~mm}$. Cross-head speed used for testing was $10 \mathrm{~mm} / \mathrm{min}$. 


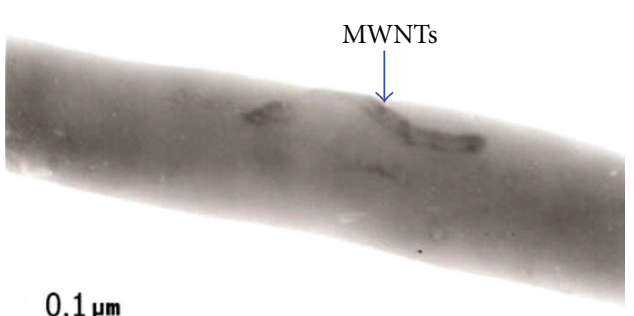

$\underline{0.1 \mu \mathrm{m}}$

(B)

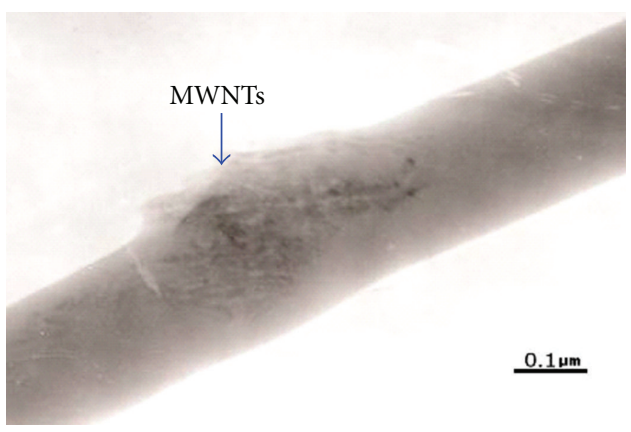

(C)



$\left(\mathrm{B}^{\prime}\right)$

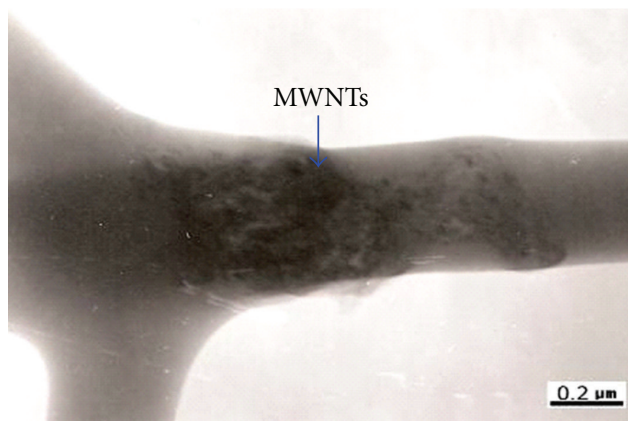

$\left(\mathrm{C}^{\prime}\right)$

Figure 2: TEM images of MWNTs/PU nanofibers without thermal treatment (B and C) and with thermal treatment $\left(\mathrm{B}^{\prime}\right.$ and $\left.\mathrm{C}^{\prime}\right)$.

\section{Results and Discussion}

3.1. Morphology. SEM analysis of the pure PU and MWNTs/PU nanofibers with and without thermal treatment prepared by electrospinning are provided in Figure 1. As can be seen the fibers diameter becomes thinner with larger MWNTs loadings. The average diameter of PU fibers was observed as $910 \pm 300 \mathrm{~nm}$. The diameters decrease to $440 \pm$ $150 \mathrm{~nm}$, and $350 \pm 90 \mathrm{~nm}$ with MWNTs concentrations increase to 1 , and $3 \mathrm{wt} . \%$, respectively. Note that MWNTs modify the spinnability of the polymer; thus, the diameter distribution and morphology of the fibers are changed. It can be deduced from the fact that there are some free electric charges within MWNTs, high MWNTs concentration leads a high surface charge density to the liquid cone, which further results a bigger acceleration to the jet flow and an increasing tensile stress to the nanofibers. So, when the other parameters are keept the same, the increasing MWNTs promote the splitting of a larger fiber into several small fibers. Therefore, the fibers become thinner and thinner as shown in Figure 1 (A, B, and C).

It can be seen from Figure $1\left(\mathrm{~A}^{\prime}, \mathrm{B}^{\prime}\right.$, and $\left.\mathrm{C}^{\prime}\right)$ that the fiber diameters were measured as $820 \pm 280 \mathrm{~nm}, 420 \pm$ $180 \mathrm{~nm}$, and $340 \pm 100 \mathrm{~nm}$, respectively. It shows that thermal treatment has little effect on the average diameters of the fibers. Without thermal treatment, the individual fibers are stacked one over another just by "contacting" mode (as shown in Figure 1 (A, B, and $\mathrm{C}$ ), and the junction point is discontinuous. After thermal treatment, the contacting fibers are bonded together at the junction point (as shown in Figure $1\left(\mathrm{~A}^{\prime}, \mathrm{B}^{\prime}\right.$, and $\left.\left.\mathrm{C}^{\prime}\right)\right)$. It means that the junction point is continuous and rigid. Therefore, we can deduce that the whole membrane forms a continuous stress transmission carrier after thermal treatment. Figure 1 also illustrates that the surface of the fibers is smooth without visible bead bars, indicating that no apparent agglomeration is produced with the formation of MWNTs/PU ultrathin fibers.

The incorporation of MWNTs into the PU nanofibers was confirmed by TEM (Figure 2). It can be visible that the incorporated MWNTs tends to align along the fiber axis when MWNTs concentration is $1 \mathrm{wt} . \%$. Some of the MWNTs are well dispersed within PU fibers or on the surface of the fibers. No significant agglomeration of MWNTs in PU fibers is observed for the samples with and without thermal treatment (Figures 2(B) and 2( $\left.\mathrm{B}^{\prime}\right)$ ). Figures 2(C) and $2\left(\mathrm{C}^{\prime}\right)$ give the TEM images of MWNTs/PU fibers with 3 wt. \% MWNTs. Those two images exhibit more MWNTs are incorporated into the fibers, agglomeration is formed with higher MWNTs loading shown in Figure $2\left(\mathrm{C}^{\prime}\right)$. It is also seen from Figure $2\left(\mathrm{C}^{\prime}\right)$ that some visible contacting point of fibers is bonded together after thermal treatment.

3.2. Thermal Properties. Figure 3 gives the TGA thermograms of all samples in this study. The pure PU, $1 \mathrm{wt} \%$ MWNTs/PU, and 3 wt.\% MWNTs/PU membranes state the temperature at which $5 \%\left(T_{5 \%}\right)$ weight loss occurs at $230^{\circ} \mathrm{C}$, $234^{\circ} \mathrm{C}$, and $240^{\circ} \mathrm{C}$, and completely decomposition around $375^{\circ} \mathrm{C}, 390^{\circ} \mathrm{C}$, and $384^{\circ} \mathrm{C}$, respectively. While, the thermal treatment membranes show nearly the same $T_{5 \%}$, but the completely decomposed temperature is $440^{\circ} \mathrm{C}$ and $466^{\circ} \mathrm{C}$ for the $2 \mathrm{wt} . \%$ and $3 \mathrm{wt} . \%$ membranes, respectively. 


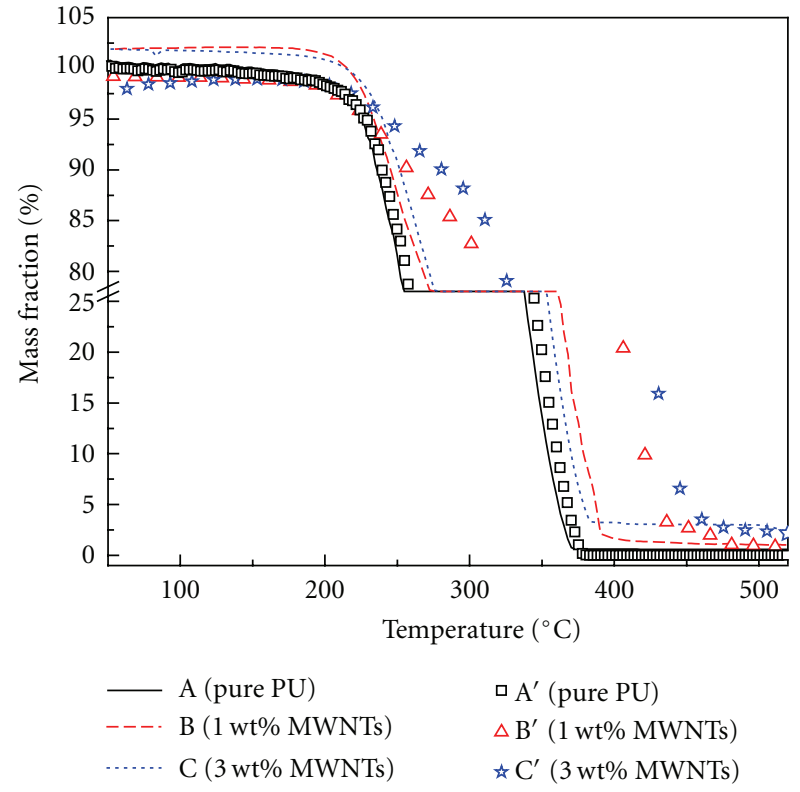

FIgURE 3: TGA curves of the studied MWNTs/PU samples.

Thus, the incorporation of MWNTs into the nanofibers can decrease the decomposition speed of the PU, but the thermal treatment has slight effect on the degradation. The actual content of the MWNTs inside the membranes can be determined using TGA curves of Figure 3. Residues of the MWNTs were analyzed at $500^{\circ} \mathrm{C}$ because $\mathrm{PU}$ and MWNTs are decomposed at approximately 240 and $800^{\circ} \mathrm{C}$, respectively. As shown in Figure 3 and Table 1, the contents of MWNTs are slightly changed, suggesting that the MWNTs in the polymer solution are successfully embedded into the nanofibers during the electrospinning process.

Figure 4(a) shows the DMA plots of storage modulus versus temperature as a function of loading MWNTs. The modulus drop is credited to an energy dissipation phenomenon involving cooperative motions of the polymer chain. The storage modulus steadily increases with higher MWNTs weight percents. The addition of 1 and $3 \mathrm{wt} . \%$ of MWNTs yields a $96 \%$ and $160 \%$ increase of the storage modulus at $-70^{\circ} \mathrm{C}$, respectively. The high-aspect ratio and elastic modulus of MWNTs greatly increase the storage modulus with higher amounts of MWNTs. It is also seen from Figure 4(a) that the storage modulus of the membranes, for example, $\mathrm{B}^{\prime}$ and $\mathrm{C}^{\prime}$, increases by nearly $100 \%$ as that of the untreated materials. This is because more MWNTs contact with each other after thermal treatment, resulting in a more rigid membrane. But the pure PU membrane is slightly affected by the thermal treatment. The loss factor curves, $\tan \delta$, of the studied samples measured by DMA are also shown in Figure 4(b). The peak height of loss factor decreases with higher MWNTs content, but the temperature determined from the peak position of $\tan \delta, T_{\mathrm{g}}$, increases to -23 and $-18^{\circ} \mathrm{C}$ for the 1 and $3 \mathrm{wt} . \%$ MWNTs loading membranes (B and C), respectively, indicating a $13 \%$ and $32 \%$ increase of the $T_{\mathrm{g}}$. While, the $T_{\mathrm{g}}$ is further improved by $17 \%$ and $21 \%\left(\mathrm{~B}^{\prime}\right.$ and $\left.\mathrm{C}^{\prime}\right)$ compared with the untreated

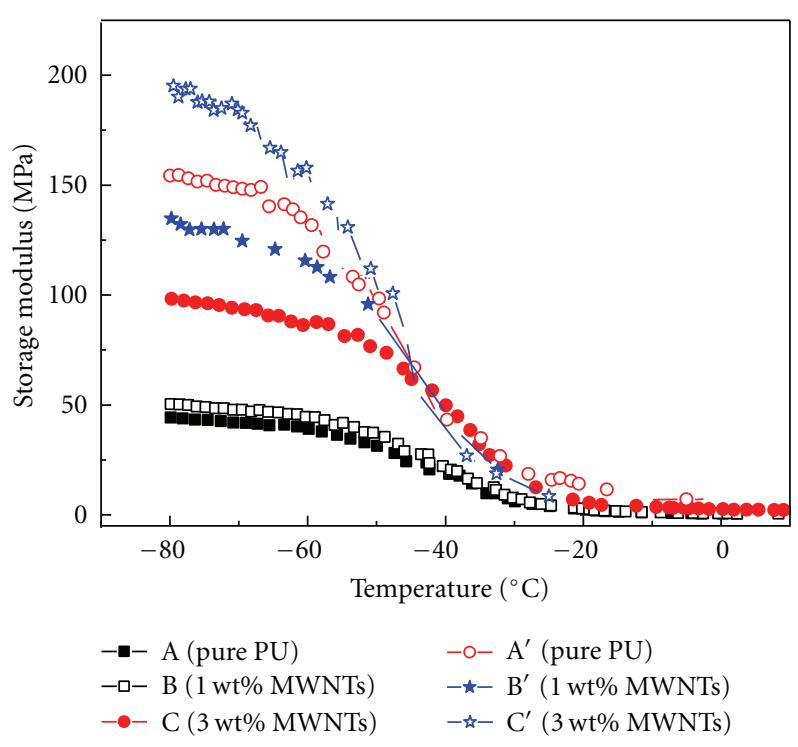

(a)

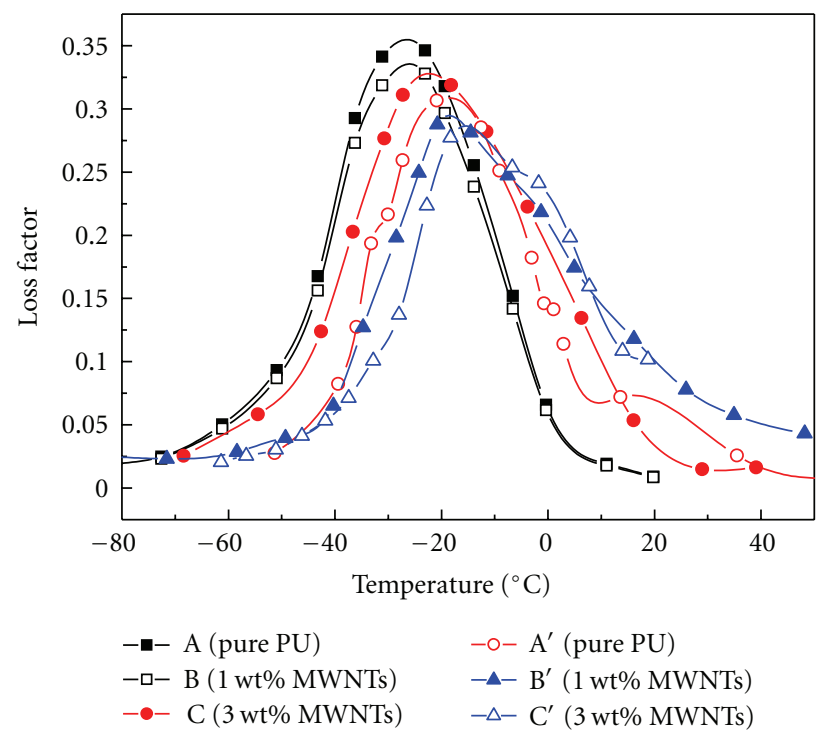

(b)

FIgure 4: DMA curves of the studied MWNTs/PU samples. (a) is storage modulus and (b) is loss factor.

materials (B and C). It also exhibits thermal treatment has slight effect on the pure PU membrane. In addition, the width $\tan \delta$ increases with higher MWNTs contents.

3.3. Conductive Properties. DC conductivity of electrospinning membranes with and without thermal treatment was measured for the studied samples (shown in Figure 5). All the experiments were run in similar conditions, giving repeatability with a deviation of less than $10 \%$ with respect to the average conductivity. The conductivity of the nanofibrous membranes increases from $2.49 \times 10^{-14} \mathrm{~S} / \mathrm{cm}$ to $4.69 \times 10^{-11}$ and $7.93 \times 10^{-8} \mathrm{~S} / \mathrm{cm}$ with the MWNTs loading increasing from $0 \mathrm{wt} . \%$ to 1 and $3 \mathrm{wt} . \%$, that is, 


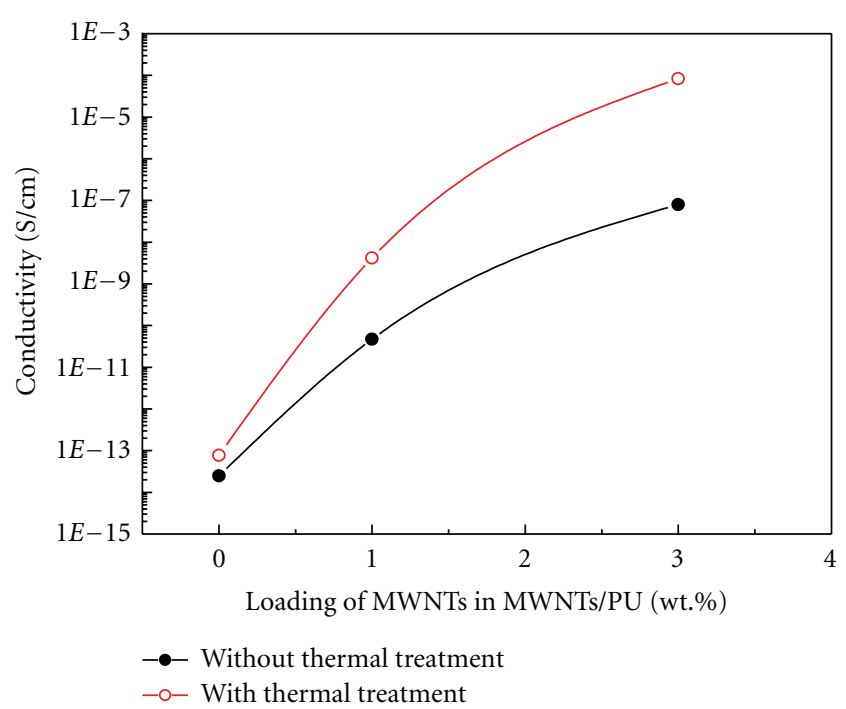

FIGURE 5: DC Conductivity of nanofibrous membranes with and without thermal treatment.

3 and 6 orders of magnitude greater than that of neat PU membrane, respectively. While, DC conductivity of the thermal treating membranes increases to $4.12 \times 10^{-9}$ and $8.29 \times 10^{-5} \mathrm{~S} / \mathrm{cm}$ for $\mathrm{B}^{\prime}$ and $\mathrm{C}^{\prime}$ samples, respectively. It states that the conductivity is improved by 2 to 3 orders of magnitude greater than that of the untreated MWNTs/PU membranes. It could be deduced that an interconnected network of MWNTs exists within in fibers; an outerconnected network of MWNTs between fibers also forms. More conductive paths result better conductivity. According to the common used classification of the electrical conducting materials, the materials with electrical conductivity lower than $10^{-6} \mathrm{~S} / \mathrm{cm}$ are treated as insulators, with electrical conductivity between $10^{-6} \mathrm{~S} / \mathrm{cm}$ and $10^{-2} \mathrm{~S} / \mathrm{cm}$ as semiconductors, and consequently with greater than $10^{-2} \mathrm{~S} / \mathrm{cm}$ as metals $[18,25]$. Thus, the membrane with MWNTs loadings of $3 \mathrm{wt} . \%\left(\mathrm{C}^{\prime}\right)$ presented in this work belongs to materials characterized as semiconductors. This finding indicates the porous membrane with $3 \mathrm{wt} . \%$ of MWNTs loadings after thermal treatment can be utilized for electrostatic discharge application.

Figure 6 illustrates the frequency dependence of the AC conductivity of MWNTs/PU membranes with up to $3 \mathrm{wt} . \%$ of MWNTs measured at room temperature. Depending on the nanotubes concentration, two distinct behaviors are observed. For the pure PU membranes $\left(\mathrm{A}\right.$ and $\left.\mathrm{A}^{\prime}\right)$, as well as for the $1 \mathrm{wt} . \%$ MWNTs/PU membranes (B and $\mathrm{B}^{\prime}$ ), AC conductivity increases linearly with the frequency, showing a typical behavior for dielectric materials. On the contrary, the $3 \mathrm{wt} . \%$ MWNTs sample (C, without thermal treatment) exhibits a small DC conductivity plateau $(40-260 \mathrm{~Hz})$, where the conductivity is independent of frequency, above which the conductivity is linearly increased again. This means the material begins to translate from dielectric to conductive materials. While, the thermal-treated sample $\left(\mathrm{C}^{\prime}\right)$ shows a better conductivity and with a lower slope than that of

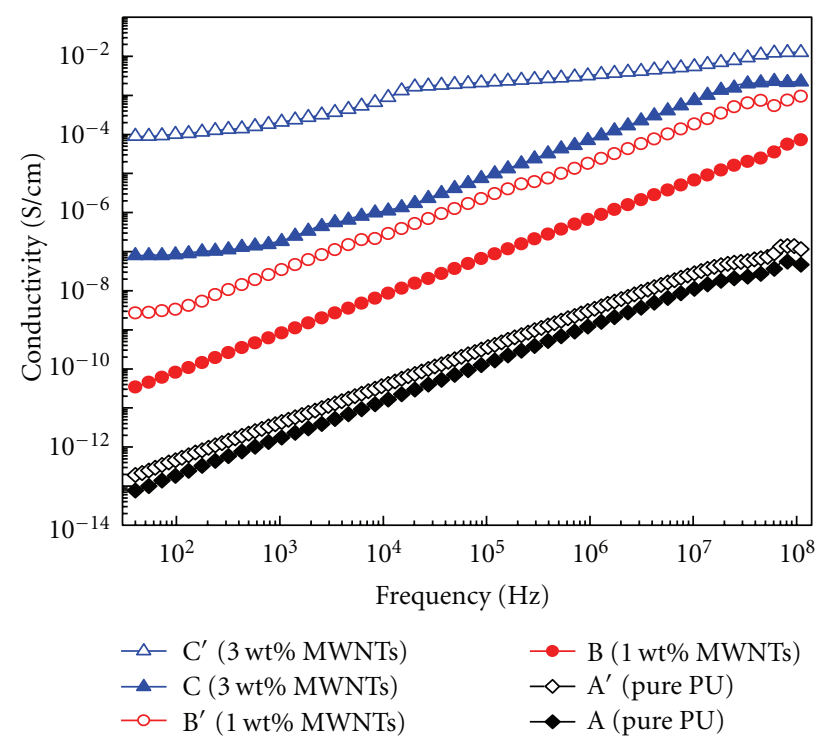

FIGURE 6: AC Conductivity of nanofibrous membranes with and without thermal treatment versus the frequency.

the C. The conductivity of the polymer/conductive-filler composites originates from two mechanisms: (1) Ohmic conduction, through direct contact of conductive fillers, and (2) non-Ohmic conduction, through the barrier-tunnelling effect between the conductive fillers separated by a polymer layer [25]. So, Ohmic conduction is easier in thermal-treated membranes than in untreated ones, which results in a higher conductivity.

3.4. Mechanical Properties. Typical stress-strain curves for pure PU (A), 1 wt.\% MWNTs/PU (B), and 3 wt.\% MWNTs/PU (C) membranes are given in Figure 7. The untreated membranes exhibit a nonlinear elastic behavior in the low-stress region $(0-2 \mathrm{MPa})$, while the thermal-treated membranes indicate linear behavior in the whole stress region. The tensile strength of $\mathrm{A}, \mathrm{B}, \mathrm{C}, \mathrm{A}^{\prime}, \mathrm{B}^{\prime}$, and $\mathrm{C}^{\prime}$ is $7.4,11.1,14.3,11.5,16.9$, and $23.8 \mathrm{MPa}$, respectively (as shown in Figures 7 and 8(a)). Compared to the sample A, the tensile strengths of the $\mathrm{B}$ and $\mathrm{C}$ composites are improved by 50 and $92 \%$, respectively. This enhancement in the mechanical properties is evidence of efficient load transfer to the MWNTs in the composite membranes. Comparing $\mathrm{A}^{\prime}$ and $\mathrm{A}, \mathrm{B}^{\prime}$ and $\mathrm{B}, \mathrm{C}^{\prime}$ and $\mathrm{C}$, the tensile strength of the former is increased by $50-60 \%$ from that of the latter (Figure 8(a)). It indicates the proposed thermal treatment method can efficiently improve the mechanical properties.

A similar trend is seen for the tensile tangent modulus. As can be seen in Figure 8(b), the thermal treatment causes an increase in modulus compared to the untreated membranes. The modulus of the pure thermal-treated PU membrane is improved by $47 \%$ from 3.2 to $4.7 \mathrm{MPa}$, and the $1 \mathrm{wt} . \%$ MWNTs/PU $\left(\mathrm{B}^{\prime}\right)$ is improved by $95 \%$ compared to the untreated B sample. As well as, the thermal treatment of $\mathrm{C}^{\prime}$ causes an increase of $143 \%$ in modulus as that of the C 


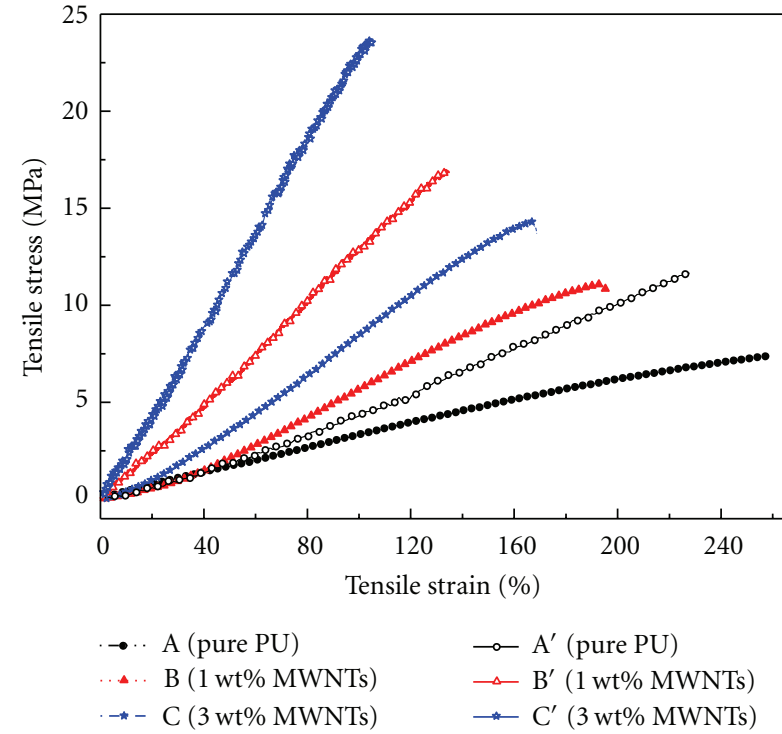

FIgURE 7: Tensile stress-strain curves of MWNTs/PUnanofibrous membranes.

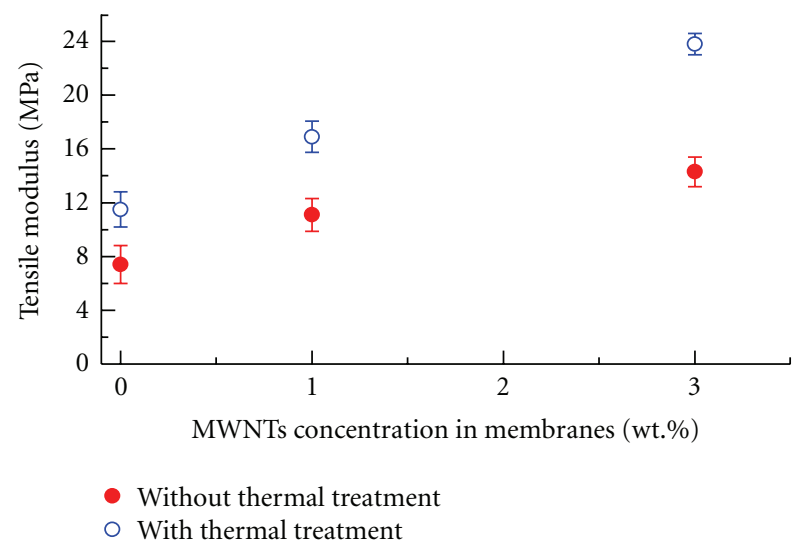

(a)

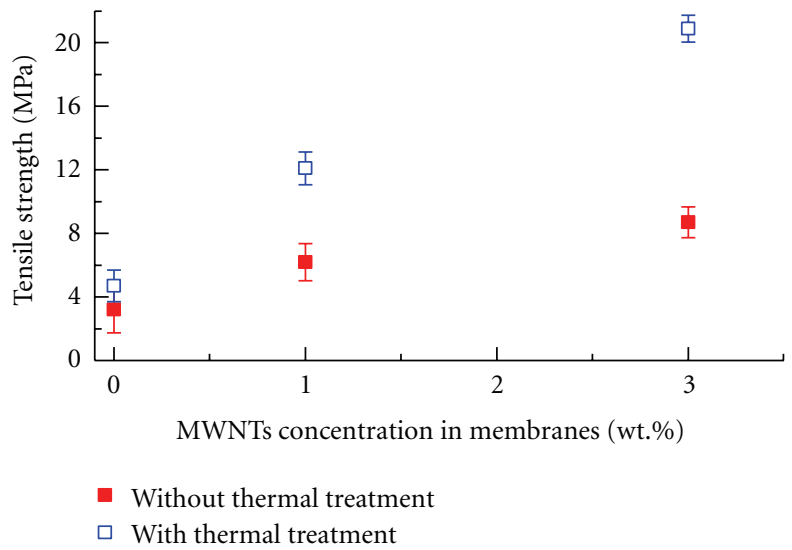

(b)

Figure 8: Tensile strength (a) and modulus (b) of the membranes with various MWNTs concentration. sample. On the contrary, the elongation at break becomes smaller by blending MWNTs and thermal treatments.

It could be deduced that the fibers in the membrane are randomly oriented, which can be classified into three main events. Fibers oriented parallel to the direction of applied strain will be stretched and bear the load. Fibers oriented at smaller angle with respect to the direction of applied strain will be rotated and then be stretched to carry some loads. Fibers oriented at larger angle with the direction of the applied strain as well as approximately perpendicular to the direction of applied strain will be buckled. The stretched fibers contribute a large-part stress in the membranes, while the buckling fibers are considered to contribute zero to the total stress in the membrane. For the untreated membranes such as $A, B$, and $C$, the fibers within the membranes are individual, and there is no continuous stress transfer between the stocking fibers (see also Figure 1 (A, B, and C). So, they mainly undergo the above-mentioned three events. On the other hand, the thermal-treated samples, that is, $\mathrm{A}^{\prime}, \mathrm{B}^{\prime}$, and $\mathrm{C}^{\prime}$, the fibers in the membranes are rigidly connected and form a continuous stress transfer carrier, as well as more MWNTs connect with each other, which results in a more efficient transfer of the stress in the membranes. Also, some fibers oriented at larger angle with respect to the direction of applied strain will be rotated and stretched because the two ends of those fibers are bonding with other fibers. Therefore, more fibers will contribute a force to the membranes for the case with thermal treatment.

\section{Conclusions}

Electrospinning technique was used to produce MWNTs/PU membranes with MWNTs concentration varying from 0,1 to $3 \mathrm{wt} . \%$. A thermal treatment method was applied to all the samples. This study indicates the thermal treatment contributes to form a continuous web of MWNTs/PU fibers. More fibers are rigidly connected with each other, as well as more MWNTs connect together, which results in a continuous stress transmission carrier and forms more conductive paths. Therefore, the storage modulus, tensile strength and modulus, and conductivity are enhanced significantly. In conclusion, we have demonstrated the feasibility of using the thermal treatment method to improve the comprehensive properties of the MWNTs/PU membranes.

\section{Acknowledgments}

The authors would like to acknowledge that the project is supported by the Natural Science Foundation of Shanghai, China (Grant no. 10ZR1431400).

\section{References}

[1] I. H. Chen, C. C. Wang, and C. Y. Chen, "Fabrication and structural characterization of polyacrylonitrile and carbon nanofibers containing plasma-modified carbon nanotubes by electrospinning," The Journal of Physical Chemistry C, vol. 114, no. 32, pp. 13532-13539, 2010. 
[2] M. Kanafchian, M. Valizadeh, and A. K. Haghi, "Electrospun nanofibers with application in nanocomposites," Korean Journal of Chemical Engineering, vol. 28, no. 2, pp. 428-439, 2011.

[3] Z. M. Huang, Y. Z. Zhang, M. Kotaki, and S. Ramakrishna, "A review on polymer nanofibers by electrospinning and their applications in nanocomposites," Composites Science and Technology, vol. 63, no. 15, pp. 2223-2253, 2003.

[4] A. Frenot and I. S. Chronakis, "Polymer nanofibers assembled by electrospinning," Current Opinion in Colloid and Interface Science, vol. 8, no. 1-2, pp. 64-75, 2003.

[5] J. Ayutsede, M. Gandhi, S. Sukiraga et al., "Carbon nanotube reinforced Bombyx mori silk nanofibers by the electrospinning process," Biomacromolecules, vol. 7, no. 1, pp. 208-214, 2006.

[6] D. Kimmel, P. Slobodiam, D. Petrás, M. Zatloukal, R. Olejnik, and P. Sáha, "Polyurethane/multiwalled carbon nanotube nanowebs prepared by an electrospieeing process," Journal of Applied Polymer Science, vol. 111, no. 6, pp. 2711-2714, 2009.

[7] S. Iijima, "Helical microtubules of graphitic carbon," Nature, vol. 354, no. 6348, pp. 56-58, 1991.

[8] B. S. Lee and W. R. Yu, "PA6/MWNT nanocomposites fabricated using electrospun nanofibers containing MWNT," Macromolecular Research, vol. 18, no. 2, pp. 162-169, 2010.

[9] R. Sen, B. Zhao, D. Perea et al., "Preparation of single-walled carbon nanotube reinforced polystyrene and polyurethane nanofibers and membranes by electrospinning," Nano Letters, vol. 4, no. 3, pp. 459-464, 2004.

[10] S. D. McCullen, D. R. Stevens, W. A. Roberts, S. S. Ojha, L. I. Clarke, and R. E. Gorga, "Morphological, electrical, and mechanical characterization of electrospun nanofiber mats containing multiwalled carbon nanotubes," Macromolecules, vol. 40, no. 4, pp. 997-1003, 2007.

[11] J. S. Jeong, S. Y. Jeon, T. Y. Lee et al., "Fabrication of MWNTs/ nylon conductive composite nanofibers by electrospinning," Diamond and Related Materials, vol. 15, no. 11-12, pp. 1839$1843,2006$.

[12] S. Mazinani, A. Ajji, and C. Dubois, "Fundamental study of crystallization, orientation, and electrical conductivity of electrospun PET/carbon nanotube nanofibers," Journal of Polymer Science B, vol. 48, no. 19, pp. 2052-2064, 2010.

[13] A. Arinstein and E. Zussman, "Electrospun polymer nanofibers: mechanical and thermodynamic perspectives," Journal of Polymer Science B, vol. 49, no. 10, pp. 691-707, 2011.

[14] T. J. Longson, R. Bhowmick, C. Gu, and B. A. Cruden, "Core-shell interactions in coaxial electrospinning and impact on electrospun multiwall carbon nanotube core, poly(methyl methacrylate) shell fibers," The Journal of Physical Chemistry C, vol. 115, no. 26, pp. 12742-12750, 2011.

[15] N. Karousis, N. Tagmatarchis, and D. Tasis, "Current progress on the chemical modification of carbon nanotubes," Chemical Reviews, vol. 110, no. 9, pp. 5366-5397, 2010.

[16] T. Nakamura, T. Ohana, M. Ishihara, M. Hasegawa, and Y. Koga, "Chemical modification of single-walled carbon nanotubes with sulfur-containing functionalities," Diamond and Related Materials, vol. 16, no. 4-7, pp. 1091-1094, 2007.

[17] K. W. Kim, K. H. Lee, M. S. Khil, Y. S. Ho, and H. Y. Kim, "The effect of molecular weight and the linear velocity of drum surface on the properties of electrospun poly(ethylene terephthalate) nonwovens," Fibers and Polymers, vol. 5, no. 2, pp. 122-127, 2004.

[18] E. J. Ra, K. H. An, K. K. Kim, S. Y. Jeong, and Y. H. Lee, "Anisotropic electrical conductivity of MWCNT/PAN nanofiber paper," Chemical Physics Letters, vol. 413, no. 1-3, pp. 188-193, 2005.
[19] M. Ignatova, T. Yovcheva, A. Viraneva, G. Mekishev, N. Manolova, and I. Rashkov, "Study of charge storage in the nanofibrous poly(ethylene terephthalate) electrets prepared by electrospinning or by corona discharge method," European Polymer Journal, vol. 44, no. 7, pp. 1962-1967, 2008.

[20] S. S. Ojha, D. R. Stevens, K. Stano, T. Hoffman, L. I. Clarke, and R. E. Gorga, "Characterization of electrical and mechanical properties for coaxial nanofibers with poly(ethylene oxide) (PEO) core and multiwalled carbon nanotube/PEO sheath," Macromolecules, vol. 41, no. 7, pp. 2509-2513, 2008.

[21] W. A. Yee, M. Kotaki, Y. Liu, and X. Lu, "Morphology, polymorphism behavior and molecular orientation of electrospun poly(vinylidene fluoride) fibers," Polymer, vol. 48, no. 2, pp. 512-521, 2007.

[22] K. H. K. Chan, S. Y. Wong, W. C. Tiju, X. Li, M. Kotaki, and C. B. He, "Morphologies and electrical properties of electrospun poly[(R)-3-hydroxybutyrate-co-(R)-3-hydroxyvalerate]/multiwalled carbon nanotubes fibers," Journal of Applied Polymer Science, vol. 116, no. 2, pp. 1030-1035, 2010.

[23] F. Ko, Y. Gogotsi, A. Ali et al., "Electrospinning of continuous carbon nanotube-filled nanofiber yarns," Advanced Materials, vol. 15, no. 14, pp. 1161-1165, 2003.

[24] J. Meng, Z. Han, H. Kong et al., "Electrospun aligned nanofibrous composite of MWCNT/polyurethane to enhance vascular endothelium cells proliferation and function," Journal of Biomedical Materials Research A, vol. 95, no. 1, pp. 312-320, 2010.

[25] F. He, S. Lau, H. L. Chan, and J. Fan, "High dielectric permittivity and low percolation threshold in nanocomposites based on poly(vinylidene fluoride) and exfoliated graphite nanoplates," Advanced Materials, vol. 21, no. 6, pp. 710-715, 2009. 

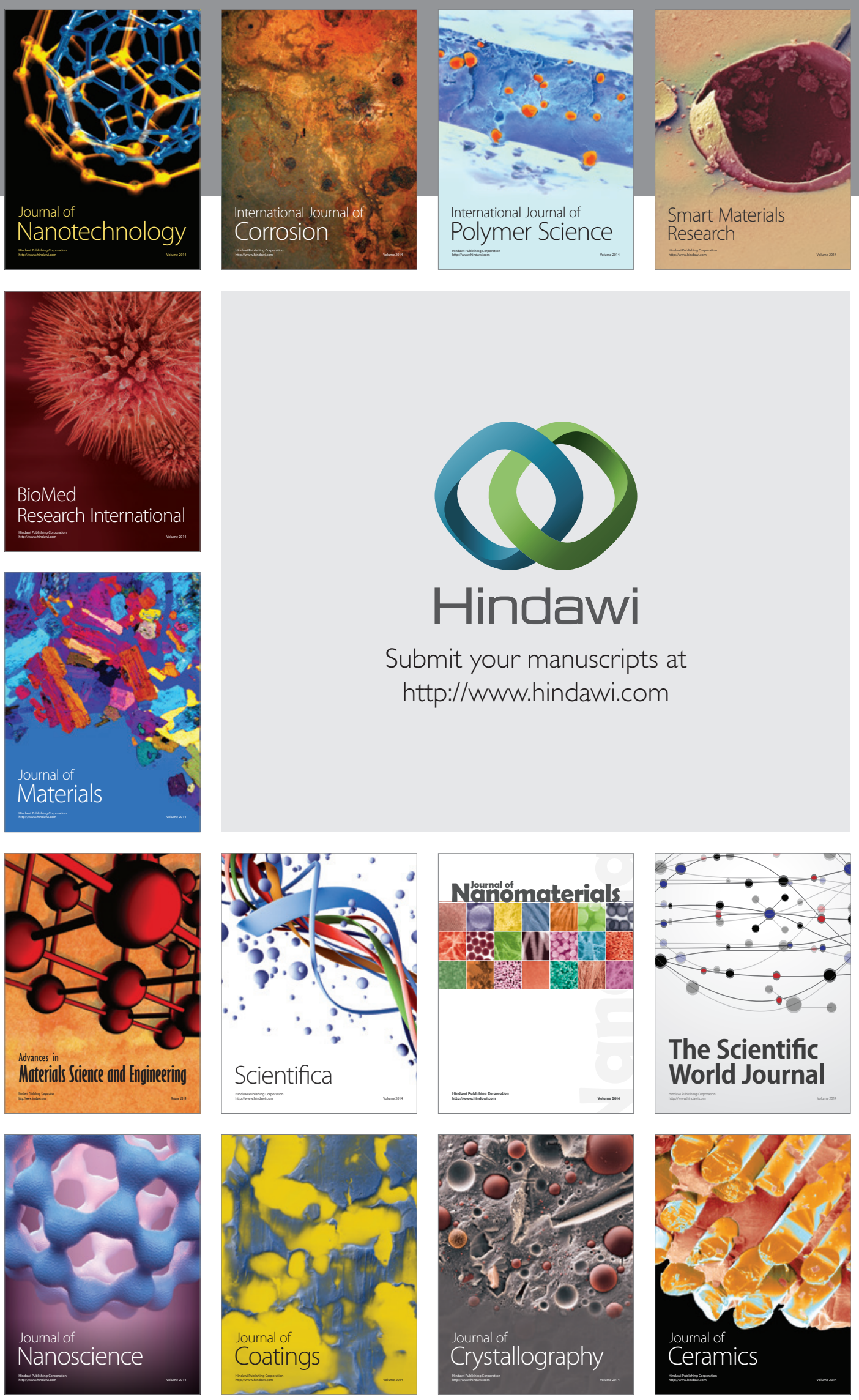

The Scientific World Journal

Submit your manuscripts at

http://www.hindawi.com

\section{World Journal}

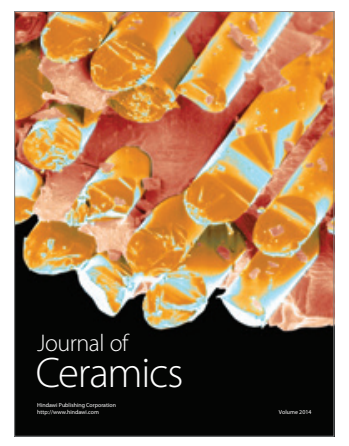

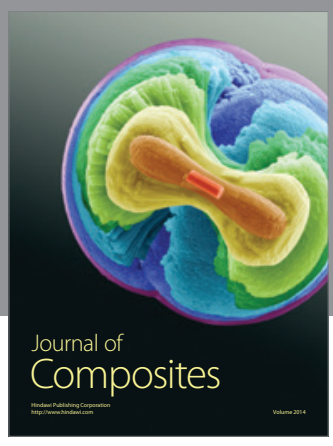
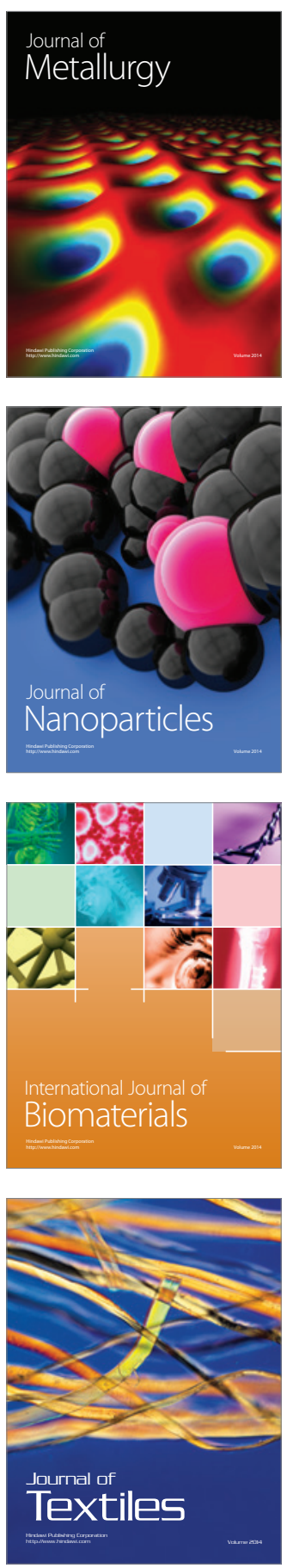